\title{
ON ESTIMATING DIFFERENTIAL CONDUCTANCE FROM NOISY I- $V$ MEASUREMENTS IN DELINEATING DEVICE PARAMETERS
}

\author{
Indrajit G. ROY \\ Spaceage Geoconsulting, 5 Bick Place, Banks, ACT 2906, Australia, \\ Tel.: +61 26294 2510, E-mail: spaceage.geocon@gmail.com
}

\begin{abstract}
Differential conductance is a key to characterizing a solid-state device. Estimation of differential conductance from current-voltage characteristic curve amounts to estimate the first order derivative from a discrete set of current-voltage measurements of the device under test. Conventional difference method in estimating derivative is inadequate when data contain noise. A robust method of estimating the first order derivative from a discrete set of evenly distributed current versus voltage measurements is designed where the method does not pose any constraints or limits on the data interval. The proposed method is formalized in the premise of the fundamental theorem of calculus and the inverse problem. The robustness of estimation is ensured using a regularization technique where the regularization operator is considered as a first order differential operator. A modified form of the L-curve technique is used to determine an optimal regularization parameter. The method is tested on synthetic and measured current-voltage characteristics of Schottky diode and MOSFET.
\end{abstract}

Keywords: differential conductance, regularization, I-V curve, solid-state device

\section{INTRODUCTION}

Characterization of solid state devices for performance analysis or modeling purposes is an essential exercise, which is, however, realized by estimating the design parameters of a device. For example, with a Schottky diode, the current-voltage $(I-V)$ characteristic curve is commonly used to estimate the diode parameters, such as the barrier height $\left(\Phi_{b}\right)$, saturation current $\left(I_{s}\right)$, ideality factor $(\eta)$, parasitic series resistance $\left(R_{S}\right)$ etc. [1-3]. In that the variation of differential conductance $(\delta G=d I / d V)$ with respect to the bias voltage is used in estimating Schottky diode parameters [4-6]. Similarly, with a metal oxide semiconductor (MOS) device the important device parameters, such as channel conductance $\left(g_{d}=d I_{D} / d V_{D}\right)$, transconductance $\left(g_{m}=d I_{D} / d V_{g}\right)$, channel mobility $\left(\mu_{0}\right)$ and threshold voltage $\left(V_{t h}\right)$ are obtained from the measurement of drain current $\left(I_{D}\right)$ versus gate-source voltage $\left(V_{g}\right)$ and the drain current $\left(I_{D}\right)$ versus drain-source voltage $\left(V_{D}\right)$ [7] . Ghibaudo [8] use drain current $\left(I_{D}\right)$ versus gate-source voltage $\left(V_{t h}\right)$ measurements to estimate the transconductance characteristic $g_{m}\left(V_{g}\right)$ which is then used in determining the channel mobility $\left(\mu_{0}\right)$ and threshold voltage $\left(V_{t h}\right)$.

Clearly, estimation of derivative from the currentvoltage measurements in a DC mode is the key for estimating parameters of a device. The current-voltage measurement of a device is relatively a simple exercise. With a programmable source meter such measurement for a device is normally carried out in small steps (or in interval) over a preset bias voltage range in an automatic fashion, and the data are readily available in a stored media. On the other hand, the exercise of estimating the differential conductance for a device, such as a Schottky diode, from the measured data often becomes non-trivial as noise in the measurements renders an unpleasant situation to an extent where the estimated conductance versus voltage characteristic curve becomes unusable for device parameter estimation.

The presence of noise in the current-voltage measure- ment for any electronic device is almost inevitable. The sources of noise are of various types, such as JohnsonNyquist noise, shot noise, flicker noise etc. Among those, the presence of Johnson-Nyquist and shot noise in the current-voltage measurements is, in fact, inevitable. Broadly, we may put those noises under the category of Gaussian distributed random noise. The strength of the noise, measured in terms of signal-to-noise ratio (SNR), often becomes the decisive factor in the exercise of parameter estimation of a device. In this paper we propose a novel technique in estimating derivatives of evenly spaced measurements in a sufficiently robust manner. We demonstrate applicability of the technique using simulated current-voltage data for a Schottky diode and MOSFET contaminated with noise, and with the real measured current-voltage data of $\mathrm{Au} / n$ GaAs Schottky diode.

\section{THEORY}

The proposed technique, based on linear inverse theory, uses the method of regularization in estimating derivative. For the sake of convenience suppose that the measurements are carried out in a forward bias mode within an interval $\left[0, V_{\text {max }}\right]$ with a step of $\Delta V$. Nevertheless, the proposed method is equally applicable for measurements in the reverse bias voltage mode as well. The evenly spaced discrete $I$ versus $V$ data, if not contaminated with noise, is assumed to form a smooth and continuously differentiable $I$ - $V$ curve. The differential conductance $\delta G$ is a function of the bias voltage and is defined as $\frac{d}{d V}[I(V)]$. Using the first principle of calculus we write

$I(V)=I(0)+\int_{0}^{V_{\text {end }}} \delta G d V$

where $I(0)$ corresponds to $V_{b}^{0}$, a zero-bias built-in voltage or a value related to band bending. Discretizing equation (1) leads to the following matrix equation

$\mathbf{A} \delta \mathbf{G}=\mathbf{I}$, 
where the matrix $\mathbf{A}$ is written as

$$
\mathbf{A}=\left[\begin{array}{cccccc}
\Delta V & 0 & 0 & \cdots & 0 & 0 \\
\Delta V & \Delta V & 0 & \cdots & 0 & 0 \\
\Delta V & \Delta V & \Delta V & \cdots & 0 & 0 \\
\vdots & \vdots & \vdots & \ddots & \Delta V & 0 \\
\Delta V & \Delta V & \Delta V & \cdots & \Delta V & \Delta V
\end{array}\right]_{N \times N}
$$

where $N$, the order of matrix $\mathbf{A}$, stands for number of samples in the dataset, $\delta \mathbf{G}$ is the vector of differential conductance and $\mathbf{I}$ is the vector comprised of measured current values corresponding to the bias voltage $V$ at the grid nodes. Truly speaking, the vector $\mathbf{I}$ is replaced with $\mathbf{I}^{\varepsilon}$ in practical situation, where $\varepsilon$ is the noise level in the measured data. To solve equation (2) we use following optimization rule

minimize $\|\hat{\mathbf{D}} \delta \tilde{\mathbf{G}}\|_{2}^{2}$ such that $\left\|\mathbf{A} \delta \tilde{\mathbf{G}}-\mathbf{I}^{\varepsilon}\right\|_{2}^{2} \leq c_{1} \varepsilon^{2}$,

where $c_{1}$ is an arbitrary constant, and

$$
\begin{aligned}
& \hat{\mathbf{D}} \delta \tilde{\mathbf{G}}= \\
& {\left[\begin{array}{cccccc}
-\Delta V^{-1} & \Delta V^{-1} & 0 & 0 & \ldots & 0 \\
0 & -\Delta V^{-1} & \Delta V^{-1} & 0 & \cdots & 0 \\
\vdots & \ddots & \ddots & \ddots & \ldots & \vdots \\
\vdots & \vdots & 0 & -\Delta V^{-1} & \Delta V^{-1} & 0 \\
0 & 0 & \cdots & 0 & -\Delta V^{-1} & \Delta V^{-1}
\end{array}\right]\left[\begin{array}{c}
\delta \tilde{G}_{1} \\
\delta \tilde{G}_{2} \\
\vdots \\
\delta \tilde{G}_{N-1} \\
\delta \tilde{G_{N}}
\end{array}\right]}
\end{aligned}
$$

where dimension of the matrix $\hat{\mathbf{D}}$ is $(N-1) \times N$ and dimension of the vector $\delta \tilde{\mathbf{G}}$ is $N \times 1$. On what follows

$$
\left(\mathbf{A}^{T} \mathbf{A}+\lambda \hat{\mathbf{D}}^{T} \hat{\mathbf{D}}\right) \delta \tilde{\mathbf{G}}=\mathbf{A}^{T} \mathbf{I}^{\varepsilon},
$$

where $\lambda$ is an arbitrary scalar, known as a regularization parameter whose optimal value, being crucial, is determined. Ideally an optimal value of regularization parameter should be chosen based on the noise level in data. Literature abounds in techniques of choosing optimal value of regularization parameters; however an optimal regularization parameter which makes a trade-off or in other words, balances the smoothness constraint and the data fitting is possibly the suitable one. Recently, selection of regularization parameter based on balancing principle is getting an increased attention [9-11]. However, as demonstrated in [11], the method requires at least an ad-hoc estimation of the noise level in the data. Unfortunately, often it is not possible to have an estimation of noise level in the measured data a priori. An intuitively appealing approach of determining an optimal value of regularization parameter is the $L$-curve technique [12-13], although the method possesses certain limitations [14]. The regularization parameter corresponding to the corner of the curve is chosen as an optimal value which is sometimes computationally challenging as, often, corner of the curve is not conspicuous to identify. We consider here a technique as suggested in [15] to determine an optimal value of the regularization parameter $\lambda_{o p}$. The discrepancy and the smoothness constraint terms corresponding to the derivative are given respectively in the following

$\Theta=\left\|\mathbf{A} \delta \hat{\mathbf{G}}-\mathbf{I}^{\varepsilon}\right\|_{2}$

and

$\Pi=\|\delta \tilde{\mathbf{G}}\|_{2}$.
We define a new function $\Psi(\lambda)$ as

$\Psi(\lambda)=\Theta \cdot \Pi$

For a set of values of $\lambda$ the plot of $\Psi(\lambda)$ versus $\lambda$ curve in a double logarithmic scale demonstrates the presence of a prominent local minimum within the chosen range of $\lambda$ values. The value of $\lambda$ corresponding to the local minimum is considered as an optimal value of the regularization parameter. Once, an optimal value of regularization parameter $\lambda_{o p}$ is estimated the robust estimate $\delta \tilde{\mathbf{G}}$ with respect to the bias voltage $V$ follows. Since an optimal value of regularization parameter $\lambda_{o p}$ satisfies the optimization rule for solving the linear set of equations with the noise contaminated data vector in the right hand side then it can be said A $\delta \tilde{\mathbf{G}}$ would provide $\tilde{\mathbf{I}}$, a best estimate of the true value of I. Hence,

$$
\left\|\tilde{\mathbf{I}}-\mathbf{I}^{\varepsilon}\right\|_{2}^{2} \approx \varepsilon^{2} .
$$

Equation (9) is used as a stopping condition and also as an appraisal of the estimated output. We use the proposed methodology in device characterization exercise.

\section{NUMERICAL EXPERIMENTS}

We conducted numerical experiment to validate the proposed methodology as a practical means of characterizing a device through the measurement of current-voltage characteristic. We conducted numerical test which was comprised of two stages. In the first stage, we simulated the I$V$ data for a device, which was contaminated with random noise and applied the proposed methodology to estimate the differential conductance. To compare the performance of the proposed method with the conventional one we also tested the noise contaminated data using a central difference scheme to estimate derivative. In the second stage we used the proposed methodology to real measured data for characterization of two types of devices viz. Schottky diode and MOSFET.

\subsection{Diode equation}

With the application of bias voltage $V$ there exists the diode current $I_{d}$ across the junction due to thermionic and/or quantum tunneling mechanisms flows. The bias voltage $V$ and the diode current $I_{d}$ are modeled with a well known diode equation based on thermionic emission theory [1] as

$I_{d}=I_{s}\left[\exp \left(\frac{q\left(V-I_{d} R_{s}\right)}{\eta \kappa_{B} T}\right)-1\right]$,

where $I_{d}$ is the measured diode current due to the applied bias voltage $V$ and $\eta$ is the ideality factor, a dimensionless parameter. The small saturation current $I_{S}$ that arises due to motion of minority carriers is given as

$I_{s}=A_{\mathrm{eff}} A^{* *} T^{2} \exp \left(-q \Phi_{b} / \kappa_{B} T\right)$,

where $A_{\text {eff }}$ is an effective area of the diode expressed in $\mathrm{cm}^{2}, A^{* *}$ is reduced Richardson constant expressed in 
$\mathrm{Acm}^{-2} \mathrm{~K}^{-2}$. For $n$-type semiconductor the value of $A^{* *}$ is $112 \mathrm{Acm}^{-2} \mathrm{~K}^{-2}$ and for $p$-type semiconductor the value of $A^{* *}$ is $32 \mathrm{Acm}^{-2} \mathrm{~K}^{-2}$ or sometimes $79 \mathrm{Acm}^{-2} \mathrm{~K}^{-2}, \Phi_{b}$ is the Schottky barrier height $(\mathrm{SBH})$ expressed generally in $\mathrm{eV}, q=1.602 \times 10^{-19} \mathrm{C}$ is the electronic charge in Coulomb, $\kappa_{B}=1.3806 \times 10^{-23} \mathrm{~J} / \mathrm{K}$ is a Boltzmann constant, $T$ is absolute temperature in Kelvin. For an ideal diode the $\operatorname{SBH}(\Phi)$ is independent of bias voltage and the ideality factor $(\eta)$ becomes unity. On what follows

$I_{d}=I_{s} \exp \left(\beta V_{d}\right)$

and

$I_{s}=A_{\mathrm{eff}} A^{* *} T^{2} \exp \left(-\beta \Phi_{b}\right)$,

where $\beta=q / \kappa_{B} T$ and $V_{d}=V-I_{d} R_{s}$. For the sake of notational simplicity we replace we replace $I_{d}$ and $V_{d}$ by $I$ and $V$ respectively. For an ideal Schottky diode the plot of $d I / d V$ versus $V$ in a semi-logarithmic scale is a straight line with a slope $d(\ln I) / d V=\beta$, The intercept $I_{s}$ made by the straight line in the $I$ axis would help in estimating the value of $\mathrm{SBH}$ and other parameters as well.

\subsection{Numerical tests with diode}

We generate the synthetic current-voltage $(I-V)$ data simulating an ideal Schottky diode with an $n$-type semiconductor, the values of parameters taken from [7] is given in Table 1 . The $I-V$ characteristic curve is generated for the forward bias voltages distributed between $0-0.2 \mathrm{~V}$ with a step of $2 \mathrm{mV}$. The synthetic data are then contaminated with additive Gaussian random noise before being implemented in the numerical test. The test was conducted with noise level of 2 and $5 \%$ respectively.

Table 1 Parameters of a Schottky diode taken from [3] used to calculate synthetic $I-V$ characteristic response

\begin{tabular}{|lll|}
\hline Parameters & Values & Units \\
\hline Barrier height $\left(\Phi_{b}\right)$ & 0.6 & $\mathrm{eV}$ \\
Area of diode $(A)$ & 0.00384 & $\mathrm{~cm}^{2}$ \\
$\begin{array}{l}\text { Reduced Richardson } \\
\text { constant }\left(A^{* *}\right)\end{array}$ & 112 & $\mathrm{Acm}{ }^{-2} \mathrm{~K}^{-2}$ \\
$\begin{array}{l}\text { Abs. temperature at } \\
25^{\circ} \mathrm{C}(\mathrm{T})\end{array}$ & 298.16 & ${ }^{\circ} \mathrm{K}$ \\
$\begin{array}{l}\text { Boltzman constant } \\
\left(\kappa_{B}\right)\end{array}$ & $1.3806 \times 10^{-19}$ & $\mathrm{~cm}^{2} \mathrm{kgs}^{-2} \mathrm{~K}^{-1}$ \\
Series reistant $\left(R_{S}\right)$ & 50 & $\Omega$ \\
\hline
\end{tabular}

The test results are presented in the panels (a)-(f) in Fig. 2. Panels (a) and (d) present current-voltage characteristics contaminated with Gaussian random noise with level 1 and $2 \%$ respectively.
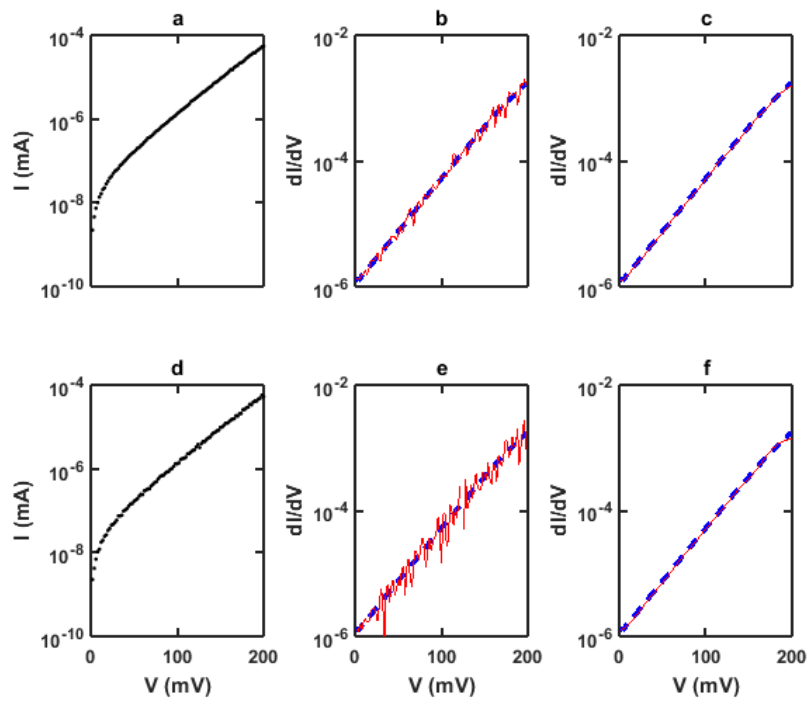

Fig. 1 Panel (a) \& (d) plots of diode current $I$ vs. forward bias voltage $V$. using parameters given in Table 1, and (b)-(f) plots of $d I / d V$ versus $V$. The upper and lower panels correspond to synthetic data contaminated by 1 , and $2 \%$ Gaussian random noise respectively. Panels (b) \& (e) are $d I / d V$ versus $V$ using difference scheme with noise (solid line) and also with no noise

(broken lines) corresponding to noise level 1 and 2\%

respectively. Panels (c) \& (f) are same as panels (b) \& (e) except that derivatives are computed using the proposed scheme (solid line). The curves with broken lines correspond to derivative with no noise in data.

Panels (b) and (e) present estimated differential conductance $(\delta G=d I / d V)$ using the central difference scheme corresponding to the Gaussian noise 1 and $2 \%$ respectively. Panels (c) and (f) present estimated differential conductance using the proposed scheme corresponding to the Gaussian noise 1 and $2 \%$ respectively. Note that broken lines (color blue) represent the analytical computed differential conductance while the solid line (color red) represents the estimated one.

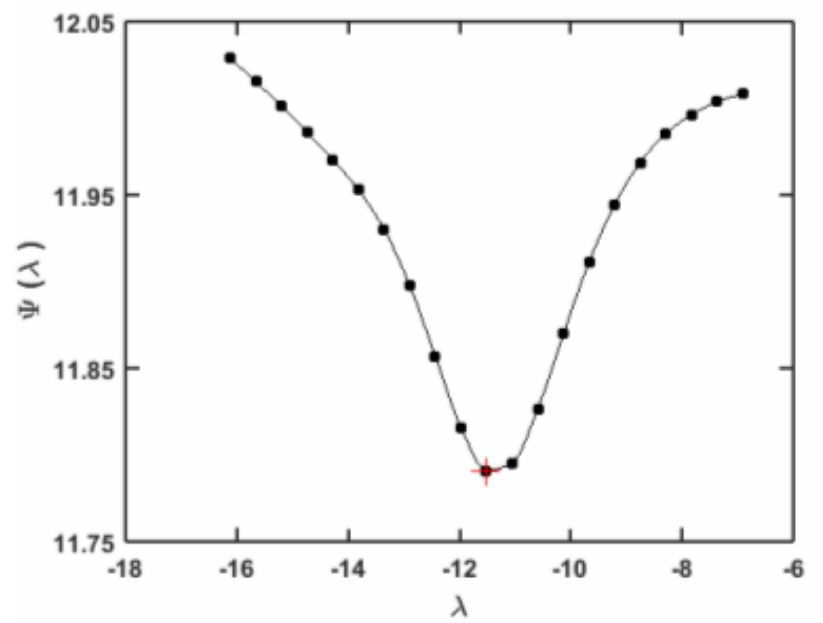

Fig. 2 Plot of $\ln [\Psi(\lambda)]$ versus $\ln (\lambda)$. The bullets are the discrete values and the solid line correspond to an interpolated curve. The location of optimum value of $\lambda$ is marked with a red colored cross mark.

Clearly, the conventional difference scheme fails to demon- 
strate sufficient robustness whereas the proposed scheme provides desired robustness in estimating the first order derivative of noisy $I-V$ measurements. In Fig. 2 we present the plot of $\Psi(\lambda)$ versus $\lambda$ in a double logarithmic scale which is used to estimate an optimal value of $\lambda$

We next consider a non-ideal Schottky diode which possesses inhomogeneous Schottky barrier Height (SBH). Variable crystallographic orientation of polycrystalline structure of metallic layer is one of the causes of inhomogeneity in SBH [16]. For a non-ideal diode the plot of $d I / d V$ versus $V$ in a semi-logarithmic scale does not demonstrate the shape of the curve as a straight line especially with a bias dependent barrier height [17]. The power exponent method has been suggested in [6] to determine Schottky diode parameters in a more generalized situation. The power exponent $\alpha$ is defined as [6]

$\alpha=\frac{d(\ln I)}{d(\ln V)}$.

The power exponent $\alpha$ is a function of the forward bias voltage $V$. The plot of $\alpha$ versus $V$ allows determining diode parameters. However, Olikh in [4] demonstrates with the synthetic data contaminated with $1 \%$ of random noise that the parameter estimation using $\alpha$ versus $V$ plot affects severely due to lack robustness in generating the $\alpha-V$ curve. In the following example we demonstrate how the proposed method provides robust estimate of $\alpha-V$ curve from noisy $I-V$ characteristic. We consider experimental data of $I-V$ curve of an $\mathrm{Au} / n \mathrm{GaAs}$ Schottky diode as used in [17]. Due to unavailability of the experimental data directly, we redrew the Fig. 2 of [17] and digitized part of the figure with a grid interval of $2 \mathrm{mV}$. The process of redrawing and digitization causes noise in the simulated experimental data. Since, our objective is to generate a smooth $\alpha-V$ curve from a noisy $I-V$ curve, this situation is ideal. The digitized I-V curve is shown in Fig. 3.

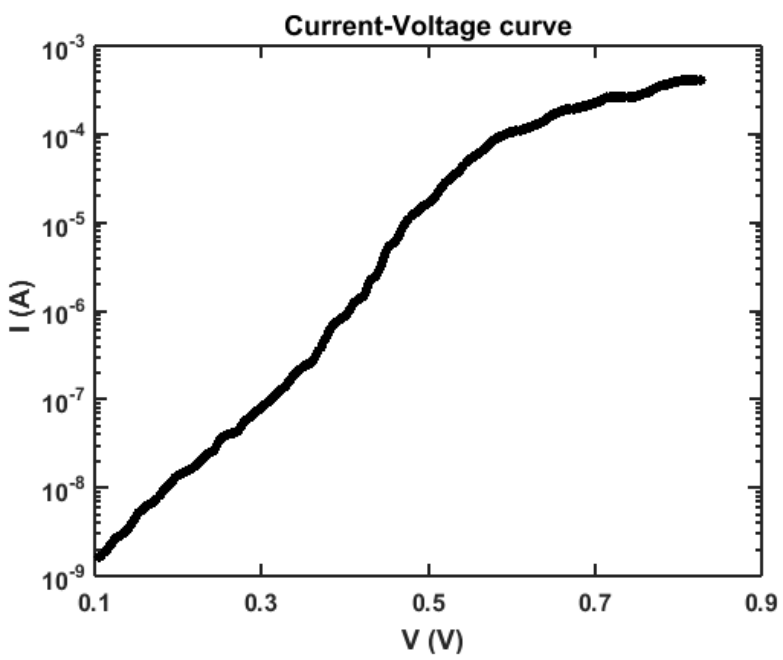

Fig. 3 Plot of $I-V$ curve of experimental data of $\mathrm{Au} / n \mathrm{GaAs}$ Schottky diode taken from [17]. The original curve of [17] was redrawn and digitized with an interval of $2 \mathrm{mV}$.

Note that the $I-V$ curve in Fig. 3 is far from being smooth. Clearly there exists noise in the data; but estimating noise level is difficult. Nonetheless, the proposed method does not require any a priori estimation of noise. We used the proposed scheme on the noisy $I-V$ data. The estimated first order derivative $d I / d V$ versus the bias voltage $V$ is plotted in Fig. 4.

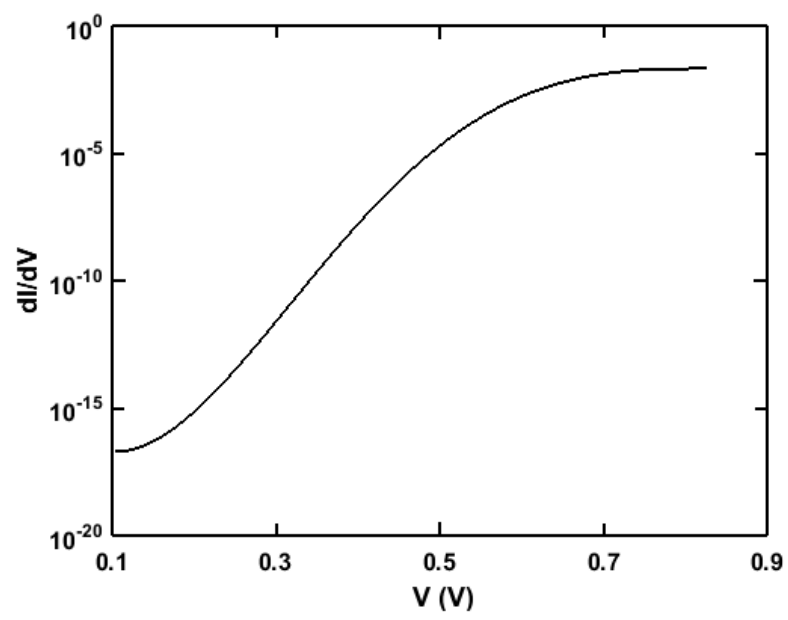

Fig. 4 Plot of $d I / d V$ versus $V$.

Note that the estimated $d I / d V$ versus $V$ curve using the proposed scheme is sufficiently smooth. In the following we present power exponent $\alpha$ versus forward bias voltage $V$ curve.

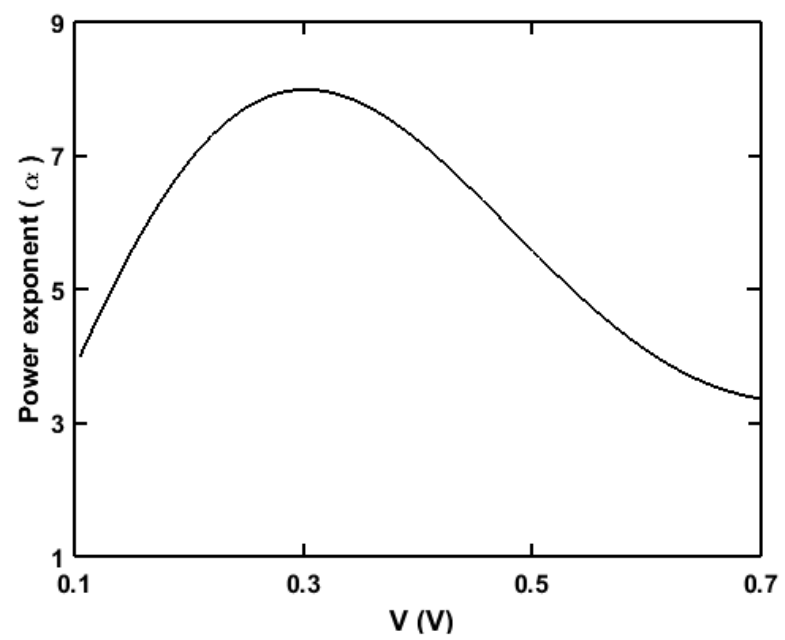

Fig. 5 Plot of $\alpha$ versus $V$ curve.

Note that the estimated power exponent $\alpha$ versus $V$ curve is sufficiently smooth which is used in estimating Schottky diode. The estimated parameter is in close agreement with the reported values. Determination of optimal value of regularization parameter $\lambda_{o p}$ is a key in the proposed scheme. The plot of $\ln [\Psi(\lambda)]$ versus $\ln (\lambda)$ in Fig. 6 demonstrates the ease in determining an optimal value of the regularization parameter, as the presence of the local minimum is quite conspicuous. 


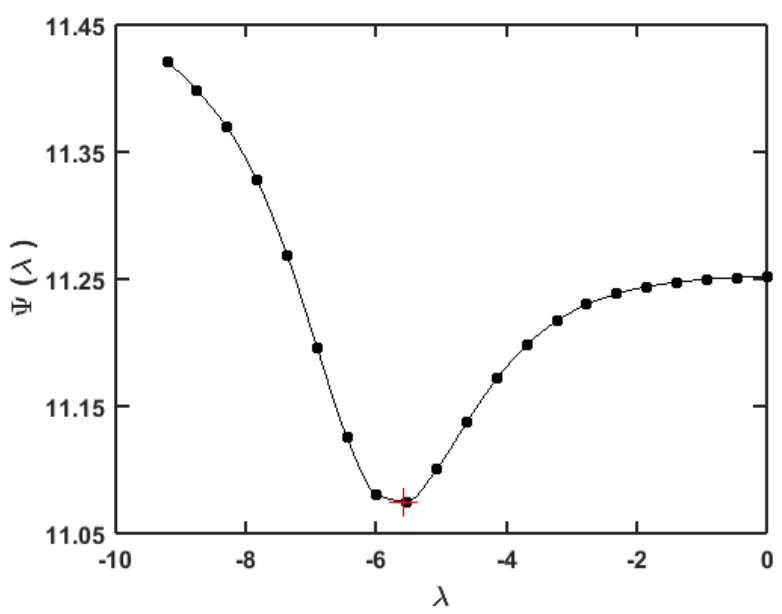

Fig. 6 Plot of $\ln [\Psi(\lambda)]$ versus $\ln (\lambda)$. The bullets are the discrete values and the solid line is interpolated one.

\subsection{Numerical tests with MOSFET}

We next conducted numerical test considering parameter extraction principle for a MOSFET device. Ghibaudo [8] demonstrates that the characteristic of drain-source current $I_{d, s}$ versus gate overdrive voltage $\left(V_{g, s}-V_{t h}\right)$ of a MOSFET can be used to estimating extract threshold voltage $V_{t h}$, low-field mobility $\mu_{0}$ and the mobility attenuation coefficient $\theta$. The revised formula for drain-source current according to [18] is given as

$\left.I_{d, s}=\mu_{\mathrm{eff}} C_{o x} \frac{W}{L} \dot{(} V_{g, s}-V_{t h}\right)\left(V_{d, s}-I_{d, s} R_{p}\right)$,

where $W$ and $L$ are the effective channel width and length respectively, $C_{o x}$ is the gate oxide capacitance, $V_{d, s}$ is the drain-source voltage, $R_{p}$ is the parasitic resistance at source and drain contacts and $\mu_{\text {eff }}$ is the effective mobility given as [19]

$\mu_{\mathrm{eff}}=\mu_{0} \frac{A_{v}\left(V_{g, s}-V_{t h}\right)^{v}}{1+\theta_{1}\left(V_{g, s}-V_{t h}\right)+\theta_{2}\left(V_{g, s}-V_{t h}\right)^{2}}$

where $\mu_{0}$ is the low field mobility, $\theta_{1}$ is the attenuation mobility factor due to phonon scattering, $\theta_{2}$ is mobility attenuation factor due to surface roughness scattering, parameter $v$ is related to Coulomb scattering due to surface roughness. $A_{v}$, the scale factor, equals to unity. The parasitic resistance $R_{p}$ is, however, neglected in the current case. The transconductance $g_{m}$ is written as

$g_{m}=\frac{d I_{d, s}}{d V_{g, s}}$

The plots of $I_{d, s} / \sqrt{g_{m}}$ and $1 / \sqrt{g_{m}}$ versus $V_{g, s}-V_{t h}$ allow to extract parameters. Using design parameters of an pMOSFET on $\mathrm{Si}(110)$ wafer (Table 2) and also equation 14 we generated synthetic drain current versus gate overdrive voltage characteristic. The synthetic data contaminated with $1 \%$ Gaussian random noise are used in the numerical test.
Table 2 Parameters of a MOSFET used to calculate synthetic $I-V$ characteristic response

\begin{tabular}{|lll|}
\hline Parameters & Values & Units \\
\hline Effective channel width $(W)$ & 20 & $\mu \mathrm{m}$ \\
Effective channel length $L$ & 7 & $\mu \mathrm{m}$ \\
Gate oxide capacitance $\left(C_{o x}\right)$ & 157 & $\mathrm{nFcm}^{-2}$ \\
Drain voltage $\left(V_{d, s}\right)$ & 200 & $\mathrm{mV}$ \\
Low field mobility $\left(\mu_{0}\right)$ & 280 & $\mathrm{~cm}^{2} / \mathrm{Vs}$ \\
Mobility attenuation factor $\left(\theta_{1}\right)$ & 0.02 & $\mathrm{~V}^{-1}$ \\
Mobility attenuation factor $\left(\theta_{2}\right)$ & 0.05 & $\mathrm{~V}^{-2}$ \\
Coulomb scattering parameter $(v)$ & 0.04 & \\
\hline
\end{tabular}

The noise contaminated $I_{d, s}$ versus gate overdrive voltage (bullets), the estimated transconductance $g_{m}$ versus gate overdrive voltage $\left(V_{g, s}-V_{t h}\right)$ using the proposed method (broken lines) and the conventional difference formula (solid line) are presented in Fig. 7. The test results clearly demonstrate the superior performance of the proposed method over the conventional one.

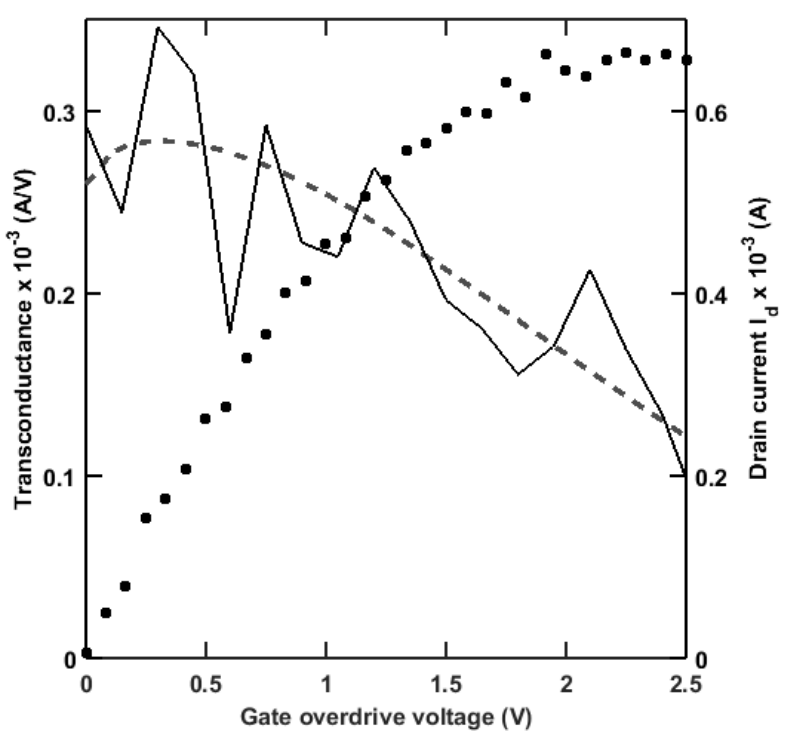

Fig. 7 Plots of synthetically generated drain current with $1 \%$ additive white Gaussian noise (bullets) the estimated transconductance using proposed scheme (gray broken lines), and using conventional difference scheme (solid line) versus gate overdrive voltage for a $p$-MOSFET on $\mathrm{Si}(110)$ wafer.

\section{CONCLUSIONS}

The robust estimate of the first order derivative of current-voltage characteristic curve is highly essential in transforming the foretold curve into conductance-voltage characteristic curve, which, in turn, allows estimating the design parameters of a solid-state device. Presence of noise in the current-voltage measurements makes the task of es- 
timating derivative remains challenging while using a conventional difference scheme. The proposed regularization based method allows robust estimation of derivative without using a priori knowledge of the noise level in the measured data. Numerical experiment with synthetic and the real measured data demonstrates the applicability of the proposed method in estimating differential conductance and transconductance of Schottky diode and MOSFET respectively.

\section{REFERENCES}

[1] SZE, S. M. - NG, K. K.: Physics of semiconductor devices, 3d ed. John Wiley \& Sons, New York, 2007.

[2] WERNER, J. H.: Schottky barrier and pn-junction I/V plotsSmall sigma evaluation, Applied Physics A 47, No. 3 (1988) 291-300.

[3] AUBRY, V. - MEYER, F.: Schottky diodes with high series resistance: Limitations of forward I-V methods, Journal of Applied Physics 76, No. 12 (1994) 79737984.

[4] OLIKH, O. Ya.: Review and test of methods for determination of the Schottky diode parameters, Journal of Applied Physics 118, No. 2 (2015) 24502.

[5] CHEUNG, S. K. - CHEUNG, N. W.: Extraction of Schottky diode parameters from forward currentvoltage characteristics, Applied Physics Letters 4 , No. 2 (1986) 85-87.

[6] MIKHELASHVILI, V. et al.: On the extraction of linear and nonlinear physical parameters in non-ideal diodes, Journal of Applied Physics 85, No. 9 (1999) 6873-6883.

[7] YTTERDAL, T. et al.: Device modeling for analog and RF CMOS circuit design, John Wiley \& Sons, New York, 2003.

[8] GHIBAUDO, G.: New method for the extraction of MOSFET parameters, Electronic Letters 24, No. 9 (1988) 543-545.

[9] LAZAROV, R. D. et al.: On the balancing principle for some problems of numerical analysis, Numerische Mathematik 106, No. 4 (2007) 659-689.

[10] ITO, K. et al.: A regularization parameter for nonsmooth Tikhonov regularization, SIAM Journal of Scientific Computing 33, No. 3 (2011) 1415-1438.

[11] ROY, I. G.: On computing first and second order derivative spectra, Journal of Computational Physics 295, No. C (2015) 307-321.
Received November 12, 2017, accepted December 20, 2017

\section{ACKNOWLEDGEMENT}

The author acknowledges an anonymous reviewer whose helps in improving the manuscript. The current research is supported by Spaceage Geoconsulting, a research oriented consulting firm.

[12] HANSEN, P. C.: Analysis of discrete ill-posed problems by means of the L-curve, SIAM Review 34, No. 4 (1992) 561-580.

[13] HANSEN, P. C.: Rank-deficient and discrete illposed problems: Numerical aspects of linear inversion. Society of Industrial and Applied Mathematics, Philadelphia, 1998.

[14] HANKE, M.: Limitations of the L-curve method in ill-posed problems, BIT Numerical Mathematics 36, No. 2 (1996) 287-301.

[15] ROY, I. G.: On robust estimation of discrete Hilbert transform of noisy data, Geophysics 78, No. 6 (2013) V239-V249.

[16] SULLIVAN, J. P. et al.: Giant variation in Schottky barrier height observed in Co/Si system, Journal of Vaccum Science and Technology B 11, No. 4 (1993) 1564-1570.

[17] MIKHELASHVILI et al.: Extraction of Schottky diode parameters with bias dependent barrier height, Solid-State Electronics 45, No. 1 (2001) 143-148.

[18] GAUBERT, P. - TERAMOTO, A.: Carrier mobility in field effect transistors, In: Different types of field effect transistors - Theory and applications, Pejovic, M. M. - Pejovic, M. M., (eds.), InTech Open, 2017.

[19] GAUBERT, P. et al.: Modeling of the hole mobility in p-channel MOS transistors fabricated on (110) oriented silicon wafers, Solid-State Electronics 54, No. 4 (2010) 420-426.

\section{BIOGRAPHY}

Indrajit G. Roy was born in 20.5.1958. He did Masters (M.Sc.Tech) degree in Applied Geophysics from Indian School of Mines, Dhanbad, India. He did PhD in Applied Geophysics from Indian School of Mines in 1987. He specializes in signal processing, computational methods in geophysics and physics and also in design of instruments. He was Senior Scientist at Geoscience Australia. Currently, he is a Principal Scientist at Spaceage Geoconsulting, a research oriented consulting firm in Australia. 